\title{
Kinematic evaluation of thoracic spinal cord sagittal diameter and the space available for cord using weight-bearing kinematic magnetic resonance imaging
}

\author{
Permsak Paholpak $\mathbb{D}^{1,2} \cdot$ Aidin Abedi $^{1} \cdot$ Rattanaporn Chamnan $^{1,3} \cdot$ Kunlavit Chantarasirirat $^{1,4} \cdot$ Koji Tamai $^{1,5} \cdot$ \\ Zorica Buser (1) $^{1} \cdot$ Jeffrey C. Wang ${ }^{1}$
}

Received: 17 June 2018 / Revised: 17 August 2018 / Accepted: 22 August 2018 / Published online: 24 September 2018

(c) International Spinal Cord Society 2018

\section{Structured abstract}

Study design Retrospective kinematic MRI (kMRI) study.

Objective To evaluate the dynamic changes of thoracic anterior and posterior space available for cord (SAC), and thoracic spinal cord in the dural sac in three positions.

Setting Expert MRI, Bellflower California; and University of Southern California, in Los Angeles, USA.

Methods A total of 118 patients ( 66 males and 52 females, mean age ( \pm SD) of $45.6 \pm 10.6$ years) who underwent thoracic spine kMRI were evaluated from T4-5 to T11-12 in flexion, neutral, and extension positions. The anterior SAC, posterior SAC, and mid-sagittal thoracic cord diameter were measured at each level from T4-5 to T11-12. Inter- and intraobserver agreements were analyzed.

Results The anterior SAC was significantly narrower in flexion position compared with other positions at T8-9 to T11-12 levels $(p<0.01)$. The T8-9 level had significantly wider posterior SAC in flexion and extension positions compared with the neutral position $(p<0.005)$. However, the posterior SAC at T9-10 was narrower in extension than the neutral position $(p=$ 0.002). Thoracic spinal cord diameter significantly increased in flexion position when compared with the neutral position at T8-9, T9-10, and T11-12 levels $(p<0.005)$.

Conclusions Thoracic spinal cord had dynamic changes with positions. In flexion position, the thoracic cord at T8-9 and below tended to move anteriorly, getting closer to the vertebral body and intervertebral disc. The mid-sagittal diameter of the thoracic cord increased in flexion position at the levels below T8-9. In the presence of lesions in anterior epidural space, the risk of spinal cord compression is higher in flexion position, especially at levels below T8-9.

Zorica Buser

zbuser@usc.edu

1 Department of Orthopaedic Surgery, Keck School of Medicine, University of Southern California, Los Angeles, CA, USA

2 Department of Orthopaedics, Faculty of Medicine, Khon Kaen University, Khon Kaen, Thailand

3 Department of Orthopaedics, Faculty of Medicine, Prince of Songkla University, Songkla, Thailand

4 Department of Orthopaedics, Pranangklao Hospital, Nonthaburi, Thailand

5 Department of Orthopaedics, Osaka City University, Osaka, Japan

\section{Introduction}

Thoracic spine is considered more stable for the spinal cord compared with cervical region, because of its relative rigidity from the rib cage [1-3]. However, the thoracic region has the narrowest space available for cord (SAC) and the least spinal cord blood supply $[4,5]$. These anatomical features make the thoracic cord at risk of developing thoracic myelopathy following compression by surrounding structures.

Despite this rigidity, thoracic spine is still affected by motion-related etiologies, such as thoracic spinal cord injury without radiographic abnormalities and thoracic ossification of the posterior longitudinal ligament (OPLL) [6]. Furthermore, changes in the thoracic spinal cord cross-sectional diameter and anteroposterior diameter of the dural sac with flexion and extension have been 
reported [6]. Morita et al. evaluated the dynamic changes of thoracic spine using functional multidetector-row computed tomography (CT) [7]. Although the study found dynamic changes of thoracic spinal cord crosssectional diameter, there were several limitations, including:

non-weight-bearing imaging, inability to identify surrounding soft tissue, and lack of information on neutral position.

Magnetic resonance imaging (MRI) of the spine is a noninvasive modality for evaluation of the soft tissue structures, such as spinal cord, cerebrospinal fluid, intervertebral disc, and ligamentum flavum [8]. The kinematic MRI (kMRI) is a noninvasive technology, which allows imaging in weight-bearing positions, including neutral, flexion, and extension $[9,10]$, and is advantageous over $\mathrm{CT}$ and plain X-ray in evaluation of soft tissue structures.

We hypothesized that the dynamic changes of thoracic spinal cord and its space in the spinal canal or dural sac might be among the predisposing factors for thoracic spinal cord compression. The aim of this study was to evaluate and describe the dynamic changes of the anterior SAC, posterior $\mathrm{SAC}$, and the thoracic spinal cord in weight-bearing condition using kMRI.

\section{Materials and methods}

All patients who had undergone thoracic spine kMRI between 2010 and 2016 were included in this study. Patients with mid-sagittal T2-weighted MRI images that had good visibility from the upper endplate of T4 to the lower endplate of T12 in neutral, flexion, and extension views were included. Patients with thoracic disc herniation, OPLL, ligamentum flavum hypertrophy, ossification of the yellow ligament (OYL), congenital anomalies, inflammatory spinal diseases, spinal infections, spinal tumors, deformities, and those who had undergone thoracic spine surgery were excluded from our study.

\section{Kinematic magnetic resonance imaging}

kMRI of the thoracic spine was performed using a 0.6 Tesla MRI scanner (Upright Multi-Position, Fornar Corp., New York, NY). The MR unit employs a horizontal orientation of two opposing magnetic doughnuts, allowing patients to be scanned in a weight-bearing position. The imaging protocol included T1- and T2-weighted sagittal fast spinecho images that were obtained with the patient seated upright in neutral $\left(0^{\circ}\right)$, flexion $\left(40^{\circ}\right)$, and extension $\left(-20^{\circ}\right)$ weight-bearing positions that were measured using a flexible surface coil.

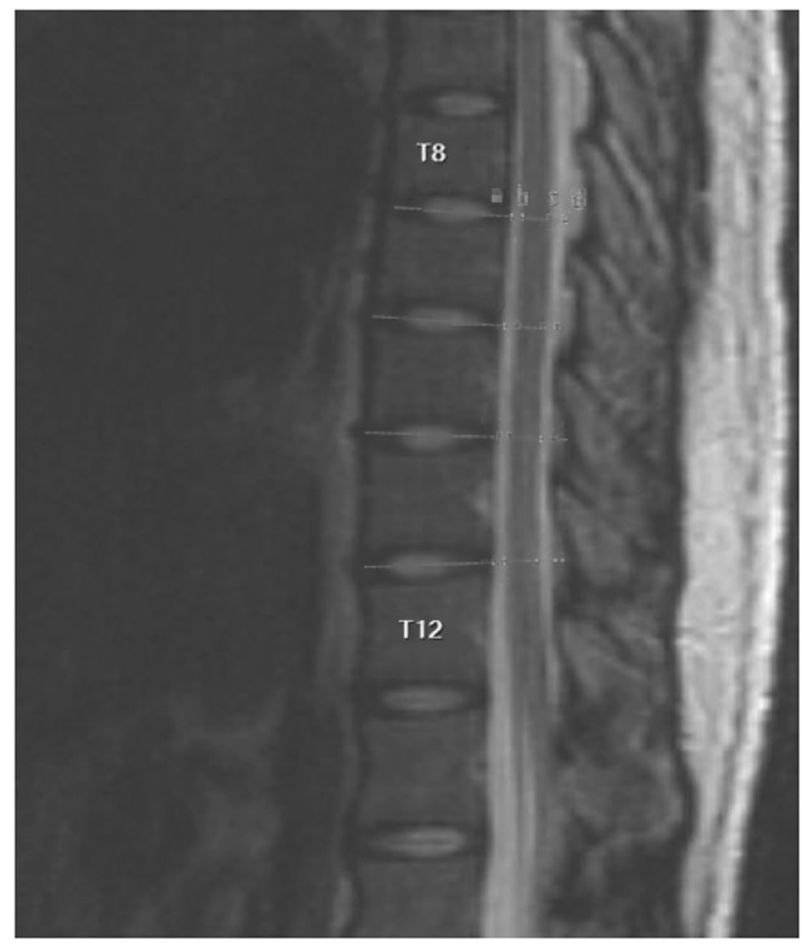

Fig. 1 Demonstration of the anterior space available for cord, posterior space available for cord, and the mid-sagittal cord diameter measurements using a magnetic resonance image. The distance between a and $\mathrm{b}$ is the anterior space available for cord, the distance between $\mathrm{b}$ and $\mathrm{c}$ is the mid-sagittal spinal cord diameter, and the distance between $\mathrm{c}$ and $\mathrm{d}$ is the posterior space available for cord

\section{KMRI analysis}

At each intervertebral disc level from T4-5 to T11-12, a line was drawn between the midpoint of the anterior disc height, the midpoint of the posterior disc height, and the corresponding point on the ligamentum flavum across the spinal canal. The anterior SAC was defined as the distance between the posterior border of the disc to the anterior border of the thoracic cord (point a to point b on Fig. 1). The posterior SAC was defined as the distance between the posterior border of the thoracic cord to the anterior border of the posterior column at each level (point $\mathrm{c}$ to point $\mathrm{d}$ on Fig. 1). The mid-sagittal cord diameter was defined as the distance between the anterior and posterior borders of thoracic cord, and was measured in the mid-sagittal plane (point $\mathrm{b}$ to point $\mathrm{c}$ on Fig. 1). All kMRI images were evaluated using eRAD PACS system software (version 7.2.38.0, eRad, South Carolina, USA).

\section{Statistical analysis}

Descriptive statistics for all parameters were performed using mean and standard deviation. Shapiro-Wilk test was used to test the normality of the data and the nonparametric 
statistics were used for analysis. Friedman's test was used to analyze the differences in SAC between neutral, flexion, and extension positions at each segment, with a $p$-value of $<0.05$ being statistically significant. The Wilcoxon-signed rank test with a Bonferroni correction was used for post-hoc analysis of the levels with a significant Friedman's test. A pvalue of 0.0167 was used as the statistical significance threshold in post-hoc analysis.

Inter- and intraobserver agreements were analyzed using 22 patients and intraclass correlation coefficients (ICCs) were calculated. The intra- and interobserver agreements for SAC was analyzed. The ICC values were assessed using the following criteria: $0-0.2$ indicated slight agreement, 0.21-0.4 indicated fair agreement, 0.41-0.6 indicated moderate agreement, $0.61-0.8$ indicated substantial agreement, and 0.81-1indicated excellent agreement [11]. All statistical analyses were performed using SPSS (Version 23.0, International Business Machines, Chicago, IL, USA).

This study was reviewed and approved by the Institutional Review Board (IRB).

\section{Results}

After filtering for the inclusion and exclusion criteria, 118 patients were included in our study. There were 66 males and 52 females, with mean age $( \pm$ SD) of $45.6 \pm 10.63$ years.

The ICCs between the two observers showed substantial to excellent agreement (range: 0.732-0.846) in all parameters.

For anterior SAC, flexion position had the narrowest SAC in all thoracic levels from T4-5 to T11-12 (Table 1). There were significant differences in anterior SAC in all three positions from T8-9 to T11-12 ( $p=0.001$ T8-9, $p<$ 0.001 at T9-10 to T11-12, Table 1). Post-hoc analysis showed that the flexion position had significantly narrower anterior SAC than neutral and extension positions at all levels from T8-9 to T11-12 $(p<0.0167)$. At T8-9 and T11-12 level, the extension position had significantly narrower anterior SAC than in neutral position $(p=0.005$ and $p=0.007$, respectively) (Table 2 ).

Assessment of the posterior SAC showed that the flexion position had the narrowest SAC at T4-5 to T6-7 levels compared with other positions, with $\mathrm{T} 4-5$ being statistically significant $(p<0.001$, Table 1$)$. From T7-8 to T11-12, posterior SAC in flexion position was wider or nearly equal to the neutral position for T7-8 $(p=0.001)$, T8-9 $(p<$ $0.001)$, and T9-10 $(p<0.001)$ (Table 1). Post-hoc analysis at T4-5 level showed that the posterior SAC in flexion was significantly narrower than in the neutral $(p<0.001)$ and extension $(p<0.001)$ positions. Whereas at the lower thoracic level, T8-9, the flexion and extension positions had a significantly wider posterior SAC than the neutral position ( $p=0.002$ and $p<0.001$, respectively). AT T9-10, flexion position showed significantly wider posterior SAC than in the extension position $(p<0.001$, Table 2$)$.

The results of the mid-sagittal thoracic spinal cord diameter in three positions are shown in Table 1. Thoracic spinal cord mid-sagittal diameter tended to be narrower in the flexion position when compared with the neutral position at T4-5 to T6-7 levels. On the other hand, the midsagittal thoracic cord diameter tended to be wider in extension compared with the neutral position at T5-6 to T7-8. At the levels below T8-9, the mid-sagittal thoracic cord diameter tended to be the widest in flexion position (Table 1). There were statistically significant differences in mid-sagittal cord diameters among three positions at T4-5 $(p=0.01)$, T6-7 $(p=0.001)$, and T8-9 to T10-11 $(p=$ 0.005 or less). The level of T11-12 showed a trend towards a statistically significant difference $(p=0.051)$. At T8-9 to T10-11, the cord diameter was significantly wider in flexion compared with the neutral position (all three levels, $p<$ 0.005 ), and extension ( $p=0.012$ or less). At T6-7, the spinal cord was significantly narrower at flexion compared with extension $(p<0.001)$ (Table 2$)$.

\section{Discussion}

The current study found that the anterior SAC, the posterior $\mathrm{SAC}$, and the mid-sagittal thoracic spinal cord diameter had dynamic changes with positional changes from neutral to flexion and extension positions.

The dynamic changes in thoracic dural sac diameter, thoracic spinal cord anteroposterior diameter, and thoracic spinal cord cross-sectional diameter between flexion and extension positions have been demonstrated using CT images [6]. However, the thoracic spinal cord compression can be caused by compression of the surrounding soft tissue structures, which cannot be identified by CT scan, such as intervertebral disc, ligamentum flavum hypertrophy or ossification, OPLL, tumor, abscess, etc. [12-16].

Although the anterior SAC, posterior SAC, and sagittal cord diameter are influenced by the degenerative pathologies of the spine in this study, we evaluated only mild spondylosis patients in order to minimize the effect of degeneration and other pathologic factors on spinal cord motion. The results from our study could help in understanding the kinematic properties of the thoracic spinal cord in normal or very mildly degenerative thoracic spine. From our results, the anterior SAC was narrower in flexion than the neutral position at T4-5 to T11-12 levels, and the thoracic sagittal spinal cord diameter was wider in flexion compared with the neutral position at the lower thoracic levels. Although the posterior SAC did not change from 
Table 1 Anterior space available for cord, posterior space available for cord, and thoracic cord sagittal diameter with statistical analysis

\begin{tabular}{|c|c|c|c|c|c|c|c|}
\hline Level & Position & $\begin{array}{l}\text { Anterior } \\
\text { SAC } \\
(\text { mean } \pm \\
\text { SD) }(\mathrm{mm})\end{array}$ & $\begin{array}{l}\text { Friedman's } \\
\text { test ( } p \text {-value) }\end{array}$ & $\begin{array}{l}\text { Posterior } \\
\text { SAC } \\
(\text { mean } \pm \\
\text { SD) }(\mathrm{mm})\end{array}$ & $\begin{array}{l}\text { Friedman's } \\
\text { test ( } p \text {-value) }\end{array}$ & $\begin{array}{l}\text { Thoracic } \\
\text { cord sagittal } \\
\text { diameter } \\
(\text { mean } \pm \mathrm{SD}) \\
(\mathrm{mm})\end{array}$ & $\begin{array}{l}\text { Friedman's } \\
\text { test ( } p \text {-value) }\end{array}$ \\
\hline \multirow[t]{3}{*}{ T4-5 } & Neutral & $1.12 \pm 1.22$ & 0.916 & $6.42 \pm 1.81$ & $<0.001 *$ & $4.54 \pm 1.13$ & $0.01 *$ \\
\hline & Flexion & $0.79 \pm 0.96$ & & $6 \pm 1.9$ & & $4.52 \pm 1.31$ & \\
\hline & Extension & $0.91 \pm 1.13$ & & $6.06 \pm 1.83$ & & $4.55 \pm 1.21$ & \\
\hline \multirow[t]{3}{*}{ T5-6 } & Neutral & $1.03 \pm 0.96$ & 0.056 & $6.45 \pm 1.9$ & 0.176 & $4.52 \pm 1.35$ & 0.675 \\
\hline & Flexion & $1.02 \pm 1.15$ & & $6.19 \pm 2.05$ & & $4.41 \pm 1.31$ & \\
\hline & Extension & $0.91 \pm 0.99$ & & $6.35 \pm 1.88$ & & $4.64 \pm 1.29$ & \\
\hline \multirow[t]{3}{*}{ T6-7 } & Neutral & $1.2 \pm 1.2$ & 0.226 & $6.1 \pm 2.11$ & 0.431 & $4.55 \pm 1.27$ & $0.001 *$ \\
\hline & Flexion & $0.98 \pm 1.26$ & & $6.06 \pm 2.1$ & & $4.36 \pm 1.21$ & \\
\hline & Extension & $1.05 \pm 1.07$ & & $6.29 \pm 1.9$ & & $4.73 \pm 1.22$ & \\
\hline \multirow[t]{3}{*}{ T7-8 } & Neutral & $\begin{array}{l}1.37 \pm \\
0.985\end{array}$ & 0.106 & $6.14 \pm 1.89$ & $0.001 *$ & $4.21 \pm 1.13$ & 0.08 \\
\hline & Flexion & $1.26 \pm 0.96$ & & $6.11 \pm 1.95$ & & $4.43 \pm 1.19$ & \\
\hline & Extension & $1.46 \pm 0.99$ & & $6.03 \pm 2.07$ & & $4.54 \pm 1.28$ & \\
\hline \multirow[t]{3}{*}{ T8-9 } & Neutral & $1.71 \pm 1.07$ & $0.001 *$ & $5.55 \pm 1.74$ & $<0.001 *$ & $4.58 \pm 1.32$ & $<0.001 *$ \\
\hline & Flexion & $1.3 \pm 0.92$ & & $5.81 \pm 2.02$ & & $4.91 \pm 1.22$ & \\
\hline & Extension & $1.56 \pm 1.01$ & & $5.81 \pm 1.94$ & & $4.47 \pm 1.23$ & \\
\hline \multirow[t]{3}{*}{ T9-10 } & Neutral & $2.19 \pm 1.24$ & $<0.001 *$ & $5.48 \pm 1.93$ & $<0.001 *$ & $4.52 \pm 1.2$ & $0.005^{*}$ \\
\hline & Flexion & $1.86 \pm 1.18$ & & $5.47 \pm 1.98$ & & $4.78 \pm 1.39$ & \\
\hline & Extension & $2.26 \pm 1.30$ & & $5.11 \pm 1.83$ & & $4.56 \pm 1.34$ & \\
\hline \multirow[t]{3}{*}{ T10-11 } & Neutral & $2.46 \pm 1.14$ & $<0.001 *$ & $4.9 \pm 1.73$ & 0.06 & $4.76 \pm 1.31$ & $0.001 *$ \\
\hline & Flexion & $2.07 \pm 1.19$ & & $5.07 \pm 2.3$ & & $4.98 \pm 1.36$ & \\
\hline & Extension & $2.33 \pm 1.54$ & & $4.57 \pm 1.99$ & & $4.96 \pm 1.57$ & \\
\hline \multirow[t]{3}{*}{ T11-12 } & Neutral & $2.63 \pm 1.49$ & $<0.001 *$ & $4.95 \pm 1.91$ & 0.836 & $5.13 \pm 1.91$ & 0.051 \\
\hline & Flexion & $2.01 \pm 1.59$ & & $4.76 \pm 1.73$ & & $6.03 \pm 1.81$ & \\
\hline & Extension & $2.35 \pm 1.52$ & & $4.87 \pm 1.99$ & & $5.7 \pm 1.77$ & \\
\hline
\end{tabular}

SAC space available for cord

*Statistically significant difference at the level of $p$-value of $<0.05$ flexion to extension at T4-5 to T6-7 levels, there was a tendency toward narrower posterior SAC in extension at lower thoracic levels. We hypothesize that in patients with thoracic disc herniation or OPLL, the severity of thoracic spinal cord compression will increase with the flexion of thoracic spine. On the other hand, in patients with myelopathic lesions such as ossified ligamentum flavum and the facet cyst, hyperextension position may deteriorate the symptoms of thoracic myelopathy, especially in the lower thoracic levels.

For the upper thoracic spine (above T8-9 level), our study found dynamic changes similar to the previous study [6], showing that the cord was narrower in flexion than in extension position. At the levels below T8-9, our results showed a trend toward wider cord diameter in flexion position compared with the neutral and extension positions, with statistically significant differences between flexion and extension at the T8-9 and T9-10 levels. These results were different from the previous study, which showed nearly similar diameters of the spinal cord at the lower thoracic levels. The differences between our findings and the previous study might be due to the weight-bearing imaging method used in this study, which is known to reveal the changes in soft tissue structures, which are not easily observed in non-weight-bearing imaging. Furthermore, these results confirmed the effect of weight bearing on visualization of the soft tissue structures in spinal column imaging.

When comparing the upper and lower thoracic levels, the lower thoracic levels (below T8-9) had more dynamic changes in most of the parameters. The explanation could be that, the levels below T7-8 are considered the transitional zone between the true thoracic ribs and false floating thoracic ribs, making the lower region more mobile [7].

The strength of this study was that we evaluated the dynamic changes of the anterior SAC, posterior SAC, and the thoracic cord in weight-bearing conditions and in all three position (neutral, flexion, and extension). The results 
Table 2 The $p$-value of the posthoc analysis at the statistically significant difference level analyzed by Friedman's test

\begin{tabular}{llllll}
\hline Anterior SAC & T8-9 & T9-10 & T10-11 & T11-12 & \\
\hline Neutral-Flexion & $<0.001^{*}$ & $0.007^{*}$ & $<0.001^{*}$ & $<0.001^{*}$ & \\
Flexion-Extension & $0.002^{*}$ & $0.002^{*}$ & $0.004^{*}$ & $<0.001^{*}$ & \\
Neutral-Extension & $0.005^{*}$ & 0.432 & 0.160 & $0.007^{*}$ & \\
Posterior SAC & $\mathrm{T} 4-5$ & $\mathrm{~T} 7-8$ & $\mathrm{~T} 8-9$ & $\mathrm{~T} 9-10$ & \\
Neutral-Flexion & $<0.001^{*}$ & 0.712 & $0.002^{*}$ & 0.907 & \\
Flexion-Extension & 0.739 & 0.072 & 0.651 & $<0.001^{*}$ & \\
Neutral-Extension & $<0.001^{*}$ & 0.401 & $<0.001^{*}$ & $<0.001^{*}$ & \\
Thoracic cord diameter & $\mathrm{T} 4-5$ & $\mathrm{~T} 6-7$ & $\mathrm{~T} 8-9$ & $\mathrm{~T} 9-10$ & $\mathrm{~T} 10-11$ \\
Neutral-Flexion & 0.157 & 0.117 & $0.002^{*}$ & $<0.001^{*}$ & $0.004^{*}$ \\
Flexion-Extension & 0.894 & $<0.001^{*}$ & $<0.001^{*}$ & $0.012^{*}$ & 0.225 \\
Neutral-Extension & 0.781 & 0.049 & 0.072 & 0.701 & 0.024 \\
\hline
\end{tabular}

SAC space available for cord

*Statistically significant difference at the $p$-value of $<0.0167$, Wilcoxon-signed rank test with Bonferroni correction

of this study help understand the dynamic pathogenesis of thoracic spinal cord compression and also can be helpful in diagnosis of the pathologies involving the thoracic spinal cord. Moreover, the presence of these dynamic positional changes indicates that, in order to decrease the risk of postoperative deterioration of myelopathy, post-laminectomy thoracic spine flexion deformity and hyper-flexion posture should be avoided, especially in patients with thoracic disc herniation and thoracic OPLL who are at a risk of anterior compression of the thoracic cord.

This study has several limitations. The $\mathrm{T} 1$ to $\mathrm{T} 3$ thoracic vertebrae could not be evaluated because of the poor image quality. Moreover, even though weight-bearing kMRI modality was used, some patients may have had difficulty in achieving full flexion or extension, due to conditions such as obesity or pain. Despite these limitations, this study provides novel and important information about dynamic changes in anterior SAC, posterior SAC, and thoracic midsagittal cord diameter from T4-5 to T11-12 levels.

\section{Conclusions}

Anterior SAC, posterior SAC, and thoracic mid-sagittal cord diameter had dynamic changes during positional changes. The flexion and extension positions put patients with myelopathy at increased risks of thoracic spinal cord compression, especially at the lower levels.

Acknowledgements We would like to thank the One Source Medical Diagnostic LLC for providing access to the kMRI image database.

Authors contributions PP was responsible for study design; data retrieval, collection, analysis and interpretation; and manuscript preparation and revision. $\mathrm{AA}, \mathrm{RC}, \mathrm{KC}$, and $\mathrm{KT}$ were responsible for data collection and analysis, and providing feedback on this manuscript. ZB was responsible for study design, data analysis and interpretation of the result, and critical revision of the manuscript. JCW was responsible for critical revision and providing feedback on the manuscript

\section{Compliance with ethical standards}

Conflict of interest The authors declare that they have no conflict of interest. Disclosures outside of submitted work: ZB: Xenco Medical (consultancy), AO Spine (consultancy). JCW: Royalties - Biomet, Seaspine, Amedica, Synthes; Investments/options - Fziomed, Promethean, Paradigm Spine, Benvenue, Nexgen, Vertiflex, Electrocore, Surgitech, Expanding Orthopedics, Osprey, Bone Biologics, Pearldiver; Board of Directors - North American Spine Society, North Amercian Spine Foundation, AO Foundation, Cervical Spine Research Society; Editorial Boards - Spine, JAAOS, The Spine Journal, Clinical Spine Surgery, Global Spine Journal; Fellowship funding (paid to institution): AO Foundation.

\section{References}

1. Sis HL, Mannen EM, Wong BM, Cadel ES, Bouxsein ML, Anderson DE, et al. Effect of follower load on motion and stiffness of the human thoracic spine with intact rib cage. J Biomech. 2016;49:3252-9.

2. Watkins Rt, Watkins R 3rd, Williams L, Ahlbrand S, Garcia R, Karamanian, et al. Stability provided by the sternum and rib cage in the thoracic spine. Spine. 2005;30:1283-6.

3. Mannen EM, Anderson JT, Arnold PM, Friis EA. Mechanical contribution of the rib cage in the human cadaveric thoracic spine. Spine. 2015;40:E760-6.

4. Hou X, Sun C, Liu X, Liu Z, Qi Q, Guo Z, et al. Clinical features of thoracic spinal stenosis-associated myelopathy: a retrospective analysis of 427 cases. Clin Spine Surg. 2016;29:86-9.

5. Barnett GH, Hardy RW Jr., Little JR, Bay JW, Sypert GW. Thoracic spinal canal stenosis. J Neurosurg. 1987;66: 338-44.

6. Morita D, Yukawa Y, Nakashima H, Ito K, Yoshida G, Machino $\mathrm{M}$, et al. Dynamic changes in the cross-sectional area of the dural sac and spinal cord in the thoracic spine. Eur Spine J. 2017;26:64-70.

7. Morita D, Yukawa Y, Nakashima H, Ito K, Yoshida G, Machino $\mathrm{M}$, et al. Range of motion of thoracic spine in sagittal plane. Eur Spine J. 2014;23:673-8. 
8. Xiong C, Daubs MD, Scott TP, Phan KH, Suzuki A, Ruangchainikom $\mathrm{M}$, et al. Dynamic evaluation of the cervical spine and the spinal cord of symptomatic patients using a kinetic magnetic resonance imaging technique. Clin Spine Surg. 2017 Oct;30(8): E1149-E1155.

9. Ruangchainikom M, Daubs MD, Suzuki A, Hayashi T, Weintraub $\mathrm{G}$, Lee CJ, et al. Effect of cervical kyphotic deformity type on the motion characteristics and dynamic spinal cord compression. Spine. 2014;39:932-8.

10. Phan KH, Daubs MD, Kupperman AI, Scott TP, Wang JC. Kinematic analysis of diseased and adjacent segments in degenerative lumbar spondylolisthesis. Spine J. 2015;15:230-7.

11. Landis JR, Koch GG. The measurement of observer agreement for categorical data. Biometrics. 1977;33:159-74.

12. Matsumoto Y, Harimaya K, Doi T, Kawaguchi K, Okada S, Inoguchi A, et al. Clinical characteristics and surgical outcome of the symptomatic ossification of ligamentum flavum at the thoracic level with combined lumbar spinal stenosis. Arch Orthop Trauma Surg. 2012;132:465-70.

13. Aizawa T, Sato T, Tanaka Y, Ozawa H, Hoshikawa T, Ishii Y, et al. Thoracic myelopathy in Japan: epidemiological retrospective study in Miyagi Prefecture during 15 years. Tohoku J Exp Med. 2006;210:199-208.

14. Aizawa T, Sato T, Sasaki H, Kusakabe T, Morozumi N, Kokubun $\mathrm{S}$. Thoracic myelopathy caused by ossification of the ligamentum flavum: clinical features and surgical results in the Japanese population. J Neurosurg Spine. 2006;5:514-9.

15. Arce CA, Dohrmann GJ. Herniated thoracic disks. Neurol Clin. 1985;3:383-92.

16. Brown CW, Deffer PA Jr., Akmakjian J, Donaldson DH, Brugman JL. The natural history of thoracic disc herniation. Spine. 1992;17(6 Suppl):S97-102. 